\title{
Pharmacokinetics of Ruxolitinib in Patients with Atopic Dermatitis Treated With Ruxolitinib Cream: Data from Phase II and III Studies
}

\author{
Xiaohua Gong ${ }^{1} \cdot$ Xuejun Chen $^{1} \cdot$ Michael E. Kuligowski ${ }^{1} \cdot$ Xing Liu $^{1} \cdot$ Xiang Liu $^{1} \cdot$ Evan Cimino $^{1} \cdot$ Ryan McGee $^{1}$. \\ Swamy Yeleswaram ${ }^{1}$
}

Accepted: 6 May 2021 / Published online: 12 May 2021

(c) The Author(s) 2021

\begin{abstract}
Background Pathogenesis of atopic dermatitis (AD) involves the Janus kinase (JAK)/signal transducer and activator of transcription (STAT) pathway. A cream formulation of ruxolitinib, a potent selective JAK1/JAK2 inhibitor, was developed for topical delivery.

Method Pharmacokinetic data were obtained from three double-blind, vehicle-controlled studies in patients with AD: a phase II study with ruxolitinib cream $0.15 \%, 0.5 \%$, or $1.5 \%$ once daily or $1.5 \%$ twice daily (BID), and two phase III studies with $0.75 \%$ or $1.5 \%$ BID. Effects of baseline characteristics on pharmacokinetics were examined. Correlations were attempted between plasma concentrations and change in hematological parameters over time.

Results Ruxolitinib plasma concentrations at steady-state $\left(C_{\mathrm{ss}}\right)$ increased with cream strength in a less-than-dose-proportional manner. In the phase III studies, overall mean (standard deviation [SD]) $C_{\mathrm{ss}}$ after ruxolitinib cream $0.75 \%$ and $1.5 \%$ BID (23.8 [35.0] and 35.7 [55.0] nM) were a fraction of the half-maximal inhibitory concentration for thrombopoietin-stimulated phosphorylated STAT3 inhibition $(281 \mathrm{nM})$, a JAK/STAT signaling marker. Three covariates were identified for $C_{\mathrm{ss}}:$ dose, percent body surface area (\%BSA) treated, and baseline Investigator's Global Assessment score. Mean (SD) bioavailability of ruxolitinib cream $1.5 \%$ BID was $6.22 \%$ (7.66\%). There were no correlations between $C_{\mathrm{ss}}$ and any hematological changes except for a transient increase in platelets at week 2.

Conclusions Plasma ruxolitinib concentrations after treatment with topical ruxolitinib cream in patients with up to $20 \%$ BSA affected by AD are not expected to lead to systemic plasma concentrations that may be associated with adverse effects commonly associated with oral JAK inhibitors.
\end{abstract}

ClinicalTrials.gov NCT03011892; NCT03745638; NCT03745651.

\section{Key Points}

In patients with atopic dermatitis, plasma concentrations of ruxolitinib were minimal following topical application of ruxolitinib cream. Consistent with this observation, systemic adverse events commonly associated with oral JAK inhibitors were not observed.

Swamy Yeleswaram yeleswaram@incyte.com

1 Incyte Corporation, Wilmington, DE, USA

\section{Introduction}

Atopic dermatitis (also known as atopic eczema) is a chronic, recurrent, inflammatory skin condition that is characterized by itchy skin (pruritus) [1-5]. It is among the 50 most prevalent diseases worldwide [6], affecting approximately 5-10\% of adults and 10-20\% of children globally [5, 7-11]. Atopic dermatitis is associated with significant comorbidities, for example, asthma and food allergies [12-14]. Moreover, atopic dermatitis ranks second among skin and subcutaneous diseases in direct disabling consequences such as itch and social stigmatization [6], and is associated with a substantial burden on quality of life, including sleep, mental health, physical functioning, social functioning, and work productivity [15-19].

Topical treatment options for patients with atopic dermatitis typically include corticosteroids and calcineurin 
inhibitors [20], which possess moderate to high efficacy, but are associated with local and systemic adverse events (AEs) that limit their long-term use. These AEs include skin atrophy (topical corticosteroids) and burning/stinging (topical calcineurin inhibitors) [17, 21-28]. Crisaborole ointment (Eucrisa ${ }^{\circledR}$; Pfizer Inc, New York, NY, USA) [29] is a nonsteroidal phosphodiesterase-4 inhibitor that abrogates inflammation by downregulating cytokine production and inflammatory markers, including helper T-cell pathways and epidermal hyperplasia and proliferation [29]. Although crisaborole ointment, which is indicated for mild-to-moderate disease, is generally associated with fewer and mild AEs, it has relatively lower efficacy compared with topical corticosteroids and calcineurin inhibitors [17]. Moreover, none of the presently marketed external drugs for atopic dermatitis are able to control pruritus in this dermatosis in a direct and prompt manner, that is, by inhibiting the itch signal transmission. Therefore, there is an unmet need for more effective and better tolerated topical atopic dermatitis therapies that will enable longer-term use, while providing rapid and sustained relief from symptoms.

Janus kinases (JAKs), consisting of JAK1, JAK2, JAK3, and Tyk2, are intracellular signaling enzymes that act downstream of key proinflammatory cytokines, and each is known to contribute to the pathogenesis of atopic dermatitis [30-37]. Recent studies indicate that JAK mediates signaling underlying sensory nerve fiber function and may mediate pruritic effects $[38,39]$. Therefore, JAK inhibitors for topical application (e.g., delgocitinib, tofacitinib, oclatinib, and ruxolitinib) have garnered interest as potential therapeutics for atopic dermatitis [40-44].

Ruxolitinib is a selective inhibitor of JAK1 and JAK2 that is approved as an oral formulation for the treatment of patients with myeloproliferative neoplasms, including myelofibrosis, polycythemia vera, and steroid-refractory acute graft-versus-host disease [45]. A topical cream formulation of ruxolitinib phosphate (ruxolitinib cream) was evaluated for the treatment of patients with atopic dermatitis in a phase II, dose-ranging study [43, 44], and was investigated in two controlled, randomized, identically designed, phase III studies followed by a 44-week follow-up period [46].

For any topical application, it is important to determine the bioavailability (i.e., the percentage of the amount of ruxolitinib that is quantifiable in plasma against the total amount of ruxolitinib in the ruxolitinib cream that was applied onto the skin lesions) resulting from repeated drug applications, so that the systemic safety profile of this drug can be characterized [47]. This study used plasma concentrations of ruxolitinib from phase II and III studies to characterize the pharmacokinetic (PK) profiles of ruxolitinib in patients treating their atopic dermatitis with ruxolitinib cream. Also tested were potential correlations between mean steady-state plasma concentrations $\left(C_{\mathrm{ss}}\right)$ and baseline patient characteristics, as well as key efficacy and safety parameters.

\section{Methods}

\subsection{Study Design and Patients}

\subsubsection{Phase II}

A double-blind, 8-week, phase II study (ClinicalTrials.gov identifier: NCT03011892) was conducted in adult patients with atopic dermatitis randomized $(1: 1: 1: 1: 1: 1)$ to topical treatment with the following creams: ruxolitinib $0.15 \%$ once daily (QD), ruxolitinib $0.5 \% \mathrm{QD}$, ruxolitinib $1.5 \% \mathrm{QD}$, ruxolitinib $1.5 \%$ twice daily (BID), vehicle BID, or active control (triamcinolone $0.1 \%$ BID) [43, 44]. Blood samples for pharmacokinetics were obtained pre-application on week 4 of the study.

\subsubsection{Phase III}

The two identically designed vehicle-controlled, doubleblind, 8-week, phase III studies (ClinicalTrials.gov identifiers: NCT03745638 [TRuE AD1]; NCT03745651 [TRuE AD2]) were conducted in adolescent and adult patients with atopic dermatitis [46]. Patients were stratified based on their Investigator's Global Assessment (IGA) score (2 or 3) and region (North America or Europe), and randomized (2:2:1) to topical treatment with ruxolitinib $0.75 \% \mathrm{BID}$, ruxolitinib $1.5 \%$ BID, or vehicle cream. After the 8 -week efficacy study period, patients enrolled in each phase III study were given an option to proceed to a corresponding 44-week safety extension study period, in which patients initially randomized to the vehicle treatment were randomized (1:1) to topical ruxolitinib $0.75 \%$ or $1.5 \%$ cream BID, whereas those initially randomized to a ruxolitinib cream remained on the same regimen. Blood samples were collected at pre-application on week 2 ( \pm 3 days), week 4 ( \pm 3 days), and week 8 ( \pm 3 days) or end of treatment (in case of early discontinuation of study treatment) during the vehicle-controlled (VC) period, and at pre-application on visits from week 12 to 52 at every 4 weeks or end of treatment during the long-term safety extension period. If there was no untreated access area available for a PK blood sample, this was not drawn on that visit and that information was recorded.

The phase II and phase III study protocols were approved by the independent ethics committee or institutional review board at each study site, and the studies were conducted in accordance with the ethical principles 
of the Declaration of Helsinki, International Council for Harmonization E6 Good Clinical Practice consolidated guidelines, and all applicable local laws/regulations. All patients provided written informed consent before initiating treatment.

\subsection{Treatment}

Patients were instructed to apply the assigned cream as a thin film to the affected areas BID $(\sim 12 \mathrm{~h}$ apart, in the morning and in the evening, $\geq 1 \mathrm{~h}$ before bedtime). If sunscreen, makeup, or other cream had been applied to the areas to be treated, the study cream was applied after those areas were washed with mild soap and water, and patted dry. If topical anti-infection agents or other topical treatments had been used, application of the study drug was to occur $\geq 1 \mathrm{~h}$ before or after application of the other topical treatment.

For patients assigned to QD regimens of ruxolitinib cream in the phase II study, the evening application consisted of the vehicle cream (which matched the ruxolitinib cream in appearance). Treatment with the triamcinolone $0.1 \%$ cream (active control of matching appearance used in the phase II study) was limited to 4 weeks (owing to the safety considerations for this drug) and was followed by treatment with the vehicle cream for an additional 4 weeks to ensure the appropriate blinding of treatment arms. On visit days, the study treatment was applied in the clinic by the patients under the supervision of the study personnel.

The amount of ruxolitinib cream used per application was determined by weighing the tube before and after each application at the clinic on the days of visit, and an average was calculated for the VC period. The average active pharmaceutical ingredient (API) dose was derived from the average amount of ruxolitinib cream multiplied by the formulation strength. If new or expanding disease areas needed treatment during the study (per consultation with the treating physician), the study cream could be applied to these areas $(\leq 20 \%$ body surface area [BSA]) as well, and the calculations used to determine the number of tubes to be provided to the patient were revised accordingly.

At week 8 of the phase III studies, patients who entered the safety extension study period received a pre-specified number of study cream tubes depending on whether BSA involvement was $\leq 5 \%$ or $>5 \%$ in the interactive response technology-based calculations. Atopic dermatitis lesions were re-evaluated every 4 weeks to confirm/determine whether treatment was required (i.e., IGA $\geq 1$ ) or if the patient should continue/enter an observation only/no treatment cycle $(\mathrm{IGA}=0)$. Throughout the study extension period, patients were instructed to self-evaluate recurrence of lesions and treat all areas of active changes $(\leq 20 \%$ BSA); if lesions cleared between study visits, treatment was stopped 3 days after clearing and restarted at the first sign of recurrence.

\subsection{Pharmacokinetic Analyses}

Collected blood samples were processed locally, per instructions from central laboratory providers, and plasma samples were shipped to the sponsor for analysis of ruxolitinib using a validated liquid chromatography-tandem mass spectrometry assay with a linear range from 1 to $1000 \mathrm{nM}$ (phase II study) or 0.3 to $300 \mathrm{ng} / \mathrm{mL}$ (i.e., $0.979-979 \mathrm{nM}$, phase III studies). Details of the assay were reported previously [48].

The steady-state plasma concentration $\left(C_{\mathrm{ss}}\right)$ of ruxolitinib was derived as the average of all observed plasma concentrations during the $\mathrm{VC}$ period, that is, concentrations from preapplication PK samples $\left(C_{\text {trough }}\right)$ at weeks 2,4 , and 8 , if any. The bioavailability after topical administration of ruxolitinib cream was assessed, based on the following equation: $F=$ $\mathrm{CL} \times C_{\mathrm{ss}} \times 12$ /average application dose of API, where $F$, $\mathrm{CL}$, and $C_{\mathrm{ss}}$ refer to the bioavailability (\%), systemic clearance [48], and steady-state plasma concentration after topical application, respectively.

\subsection{Statistical Analyses}

The ruxolitinib $C_{\mathrm{ss}}$ was analyzed as a function of the application dose of ruxolitinib freebase (the API) using a linear model framework in which the concentration and dose data were transformed to the natural logarithm domain. Patient demographics (i.e., age, sex, race), baseline characteristics (geographical regions, \%BSA treated), and baseline disease severity (Eczema Area and Severity Index [EASI] and/or IGA score) were evaluated as potential covariates. The correlations between $C_{\mathrm{ss}}$ (by quartiles) and changes in platelet count, mean platelet volume, hemoglobin level, and absolute neutrophil count were evaluated. Descriptive summary and statistical analyses were performed using SAS software version 9.4 (SAS Institute, Inc., Cary, NC, USA). Statistical plots of data were generated using SAS or R version 3.6.1 [49].

\section{Results}

\subsection{Patients}

In the phase II study, 307 patients were enrolled and randomized, and the PK population included 188 patients who were in one of the four ruxolitinib cream treatment groups. 
For the phase II PK population, the median age was 37.0 (range 18.0-70.0) years, 53.7\% of patients were female, $59.0 \%$ were white, mean (SD) total BSA involvement was $9.54 \%(5.47 \%)$, and $69.1 \%$ of patients had an IGA score of 3 .

In the phase III studies (TRuE AD1 and TRuE AD2) combined, 1249 patients were enrolled, randomized, and treated, 1119 completed treatment in the VC period, and the PK population for the VC period included 951 patients who were in one of the two ruxolitinib cream treatment groups. For the phase III PK population, the median age was 32.0 (range 12-85) years, $61.5 \%$ of patients were female, $70.8 \%$ of patients were white; mean (SD) total BSA involvement in current atopic dermatitis episode was $9.76 \%$ (5.31\%), and $75.7 \%$ of patients had an IGA score of 3 .

In both phase II and III studies, baseline demographics and characteristics were similar across treatment groups; for further details of patient characteristics, see $[43,46]$.

\subsection{Pharmacokinetic Results}

\subsubsection{Phase II}

The baseline characteristics of \% BSA and lesion area and the PK results are shown in Table 1. In the phase II study, $\% \mathrm{BSA}$, lesion area, and product application rate are similar across the dose groups. The steady-state trough plasma concentrations $\left(C_{\text {trough }}\right)$ of ruxolitinib determined at week 4 increased with the cream strength in a less-than-doseproportional manner $(0.15 \%$ vs $0.5 \%$ QD vs $1.5 \%$ QD) (Table 1). The mean steady-state $C_{\text {trough }}$ at the highest dose of $1.5 \%$ BID was $47.7 \mathrm{nM}$. A simple linear regression slope between the $C_{\text {trough }}$ on week 4 and the average application dose of ruxolitinib API, both transformed into the natural logarithm domain, is 0.805 (90\% CI 0.658-0.951) for the three cohorts of QD regimens only (Table 2).

Table 1 Descriptive summary of baseline patient characteristics and pharmacokinetic parameters

\begin{tabular}{|c|c|c|c|c|c|c|}
\hline $\begin{array}{l}\text { Ruxolitinib } \\
\text { cream treat- } \\
\text { ment }\end{array}$ & $\begin{array}{l}\text { Num- } \\
\text { ber of } \\
\text { patients }\end{array}$ & $\begin{array}{l}\text { Percent BSA treated } \\
(\%)\end{array}$ & $\begin{array}{l}\text { Area of lesion }(\mathrm{s}) \\
\text { treated }\left(\mathrm{cm}^{2}\right)\end{array}$ & $\begin{array}{l}\text { Study drug product } \\
\text { application rate }(\mathrm{mg} / \\
\left.\mathrm{cm}^{2}\right)\end{array}$ & $\begin{array}{l}\text { Steady-state trough } \\
\text { concentration of } \\
\text { ruxolitinib }(\mathrm{nM})^{\mathrm{a}}\end{array}$ & Bioavailability (\%) \\
\hline \multicolumn{7}{|c|}{ Phase II study (NCT03011892) } \\
\hline $0.15 \% \mathrm{QD}$ & 46 & $8.91 \pm 5.34(7.75)$ & $1800 \pm 1080(1590)$ & $2.15 \pm 1.73(1.82)$ & $4.11 \pm 9.06(1.14)$ & $8.90 \pm 17.2(2.60)$ \\
\hline $0.5 \% \mathrm{QD}$ & 46 & $8.87 \pm 5.03(7.00)$ & $1780 \pm 1110(1270)$ & $2.04 \pm 1.40(1.51)$ & $9.35 \pm 15.5(3.73)$ & $6.06 \pm 7.28(4.88)$ \\
\hline $1.5 \% \mathrm{QD}$ & 49 & $9.89 \pm 6.18(8.00)$ & $1990 \pm 1300(1430)$ & $2.00 \pm 1.35(1.83)$ & $30.6 \pm 44.2(15.8)$ & $7.23 \pm 7.73(4.56)$ \\
\hline $1.5 \% \mathrm{BID}$ & 47 & $10.5 \pm 5.21(10.4)$ & $2050 \pm 1020(2010)$ & $1.92 \pm 1.95(1.36)$ & $47.7 \pm 79.6(23.1)$ & $5.68 \pm 5.58(4.17)$ \\
\hline Total & 188 & $9.54 \pm 5.47(8.00)$ & $1910 \pm 1130(1600)$ & $2.03 \pm 1.61(1.72)$ & NA & NA \\
\hline \multicolumn{7}{|c|}{ Phase III studies combined (NCT03745638 [TRuE AD1]; NCT03745651 [TRuE AD2]) (VC period only) } \\
\hline $0.75 \% \mathrm{BID}$ & 472 & $9.92 \pm 5.32(8.50)$ & $1860 \pm 1020(1610)$ & $1.44 \pm 1.04(1.22)$ & $23.8 \pm 35.0(10.9)$ & $7.68 \pm 8.88(4.75)$ \\
\hline $1.5 \% \mathrm{BID}$ & 479 & $9.60 \pm 5.30(8.00)$ & $1820 \pm 1040(1530)$ & $1.49 \pm 1.09(1.26)$ & $35.7 \pm 55.0(15.4)$ & $6.22 \pm 7.66(3.64)$ \\
\hline Total & 951 & $9.76 \pm 5.31(8.10)$ & $1840 \pm 1030(1570)$ & $1.47 \pm 1.07(1.23)$ & NA & NA \\
\hline
\end{tabular}

Values are presented as mean $\pm \mathrm{SD}$ (median)

$B I D$ twice daily, $B S A$ body surface area, NA not applicable, $Q D$ once-daily, $S D$ standard deviation, $V C$ vehicle-controlled

aPhase II study: $C_{\text {trough }}$ at week 4; phase III studies: averaged $C_{\text {trough }}$ at weeks 2, 4, and 8

Table 2 Parameter estimates of dose-PK models

\begin{tabular}{|c|c|c|c|c|c|}
\hline Analysis & Parameter & Estimate & RSE (\%) & $p$-value & $90 \% \mathrm{CI}$ \\
\hline Phase II SLR (QD only) & Exponent on API dose & 0.805 & 11.0 & $<0.0001$ & $0.658-0.951$ \\
\hline Phase III SLR (BID only) & Exponent on API dose & 0.801 & 6.4 & $<0.0001$ & $0.717-0.886$ \\
\hline \multirow[t]{4}{*}{ Phase III final model } & Exponent on API dose & 0.462 & 11.6 & $<0.0001$ & $0.356-0.567$ \\
\hline & Exponent on region $=$ Europe & 0.782 & 12.3 & $<0.0001$ & $0.594-0.970$ \\
\hline & Exponent on baseline IGA 3 & 0.322 & 31.5 & 0.0008 & $0.123-0.522$ \\
\hline & Exponent on transformed \%BSA & 0.602 & 14.0 & $<0.0001$ & $0.436-0.767$ \\
\hline
\end{tabular}

RSE $=$ standard error/estimate $\times 100(\%)$

Transformed \%BSA was derived as $\log (\% \mathrm{BSA} /$ median) in which the observed median $\% \mathrm{BSA}$ was $8.1 \%$

$A P I$ active pharmaceutical ingredient, BID twice daily, BSA body surface area, $C I$ confidence interval, IGA Investigator's Global Assessment, $Q D$ once daily, $P K$ pharmacokinetic, $R S E$ relative standard error, $S L R$ simple linear regression between log-transformed concentrations and API dose 
Application of ruxolitinib cream 1.5\% BID resulted in a less-than-proportional increase in the steady-state $C_{\text {trough }}$ compared with QD application of the same strength. The distribution of plasma concentrations around the mean was similar across the dose groups. The mean bioavailability of ruxolitinib after topical application was not dose- or strength-dependent. At 1.5\% BID, the mean topical bioavailability was $5.68 \%$.

\subsubsection{Phase III}

After BID applications of ruxolitinib cream $0.75 \%$ and $1.5 \%$, the mean (SD) $C_{\mathrm{ss}}$ of ruxolitinib were $23.8(35.0) \mathrm{nM}$ $(N=472)$ and $35.7(55.0) \mathrm{nM}(N=479)$, respectively, during the VC period in pooled data from the phase III studies of TRuE AD1 and TRuE AD2 (Table 1). The time-course of $C_{\text {trough }}$ in the phase III studies is shown in Fig. 1. The median ruxolitinib $C_{\text {trough }}$ after topical applications of both $0.75 \%$ and $1.5 \%$ BID ruxolitinib cream were stable across visits during the $\mathrm{VC}$ period, and then showed a decrease over week 12 , and was stable beyond that visit. Ruxolitinib $C_{\mathrm{ss}}$ were compared between the two regions of North America and Europe, as well as between patients with mild (baseline IGA score of 2) and moderate (baseline IGA score of 3) disease. The ruxolitinib $C_{\mathrm{ss}}$ in patients from Europe with a baseline IGA of 3 trended higher compared with patients of other strata (i.e., baseline IGA of 2 from Europe and baseline IGA of 2 or 3 from North America) (Fig. 2). A simple linear regression was applied between application dose of ruxolitinib API and $\mathrm{C}_{\mathrm{ss}}$, with both values transformed into
Fig. 1 Ruxolitinib trough concentration over time after treatment with the indicated concentrations of ruxolitinib cream in phase III studies combined. BID twice daily, LTS long-term safety, $V C$ vehiclecontrolled
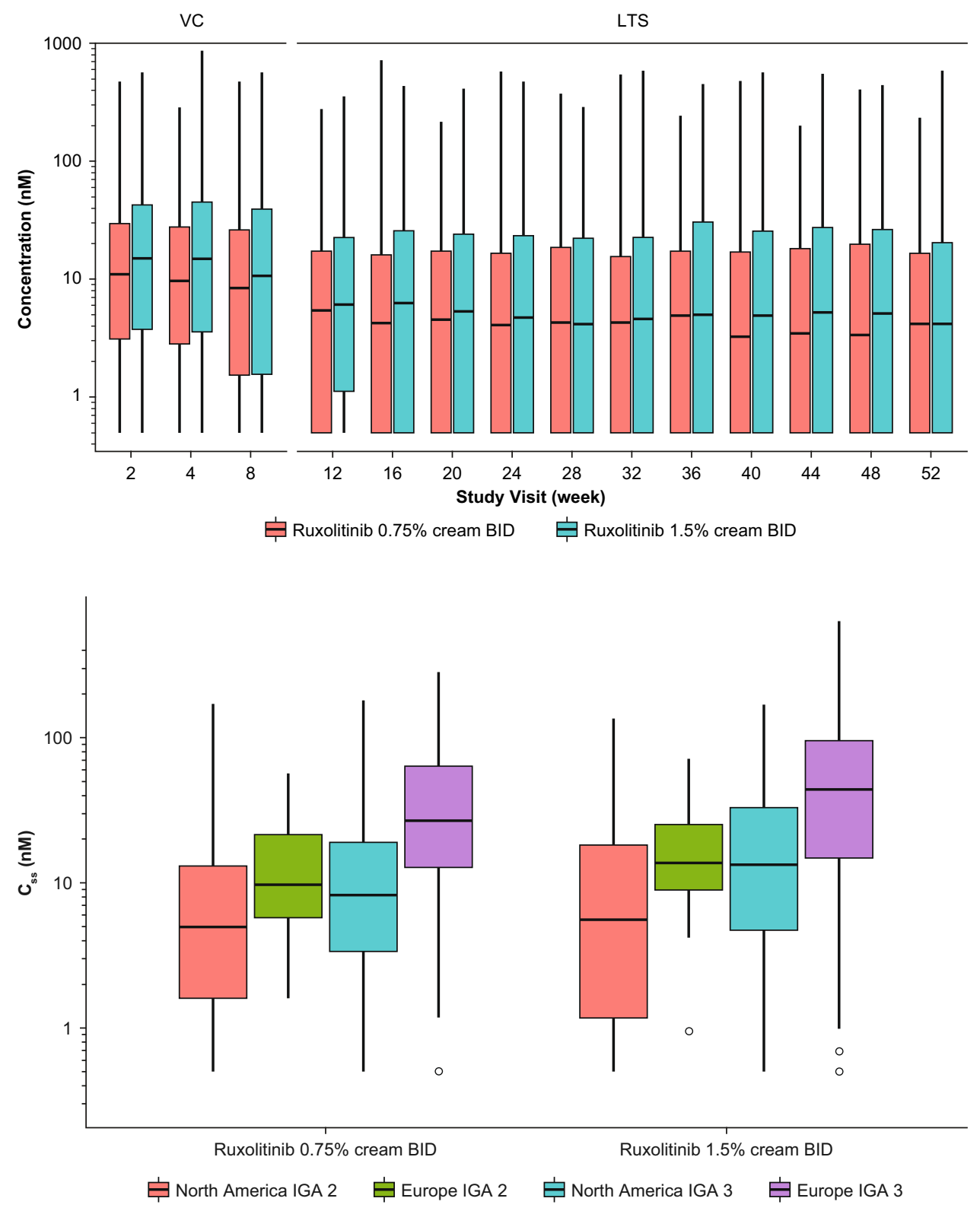

Fig. 2 Boxplots of $C_{\mathrm{ss}}$ versus actual treatment during $\mathrm{VC}$ period, stratified by geographical region and baseline IGA score, in phase III studies combined. BID twice daily, $C_{s}$ steady-state plasma concentration, IGA Investigator's Global Assessment, $V C$ vehicle-controlled 
natural logarithmic domain. The slope of the regression line was 0.801 (90\% CI 0.717-0.886) (Table 2).

Impacts of covariates on the ruxolitinib $C_{\mathrm{ss}}$ in phase III studies were evaluated using a number of potential variables such as study, demographics (sex, age, race, ethnicity, and body surface area), baseline disease severity such as \%BSA involvement with atopic dermatitis, treated lesion area, and EASI score. The clinical study design factors such as stratifications by IGA score (2 vs 3 ) and region (North America vs Europe) were also evaluated. The exploratory scatterplots

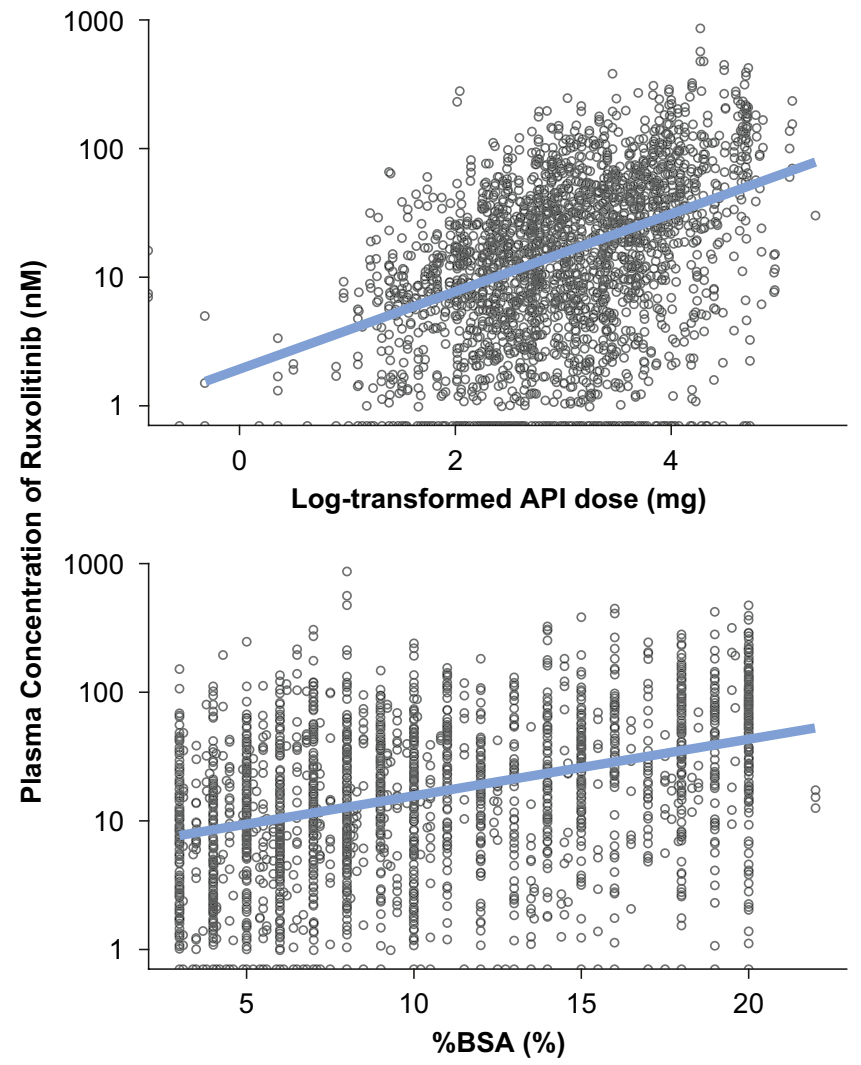

for dose, EASI score, \%BSA treated, and age are shown in Fig. 3. A trend line with positive slope was observed for dose, \%BSA, and EASI score, whereas there was no such trend for the correlation with age.

The final dose-PK model includes the study design factors of geographical region (North America vs Europe) and baseline IGA score ( 2 vs 3 ), and the continuous covariate of \% BSA involvement with atopic dermatitis at baseline (i.e., the \%BSA treated during the VC period) as significant covariates and the parameter estimates for

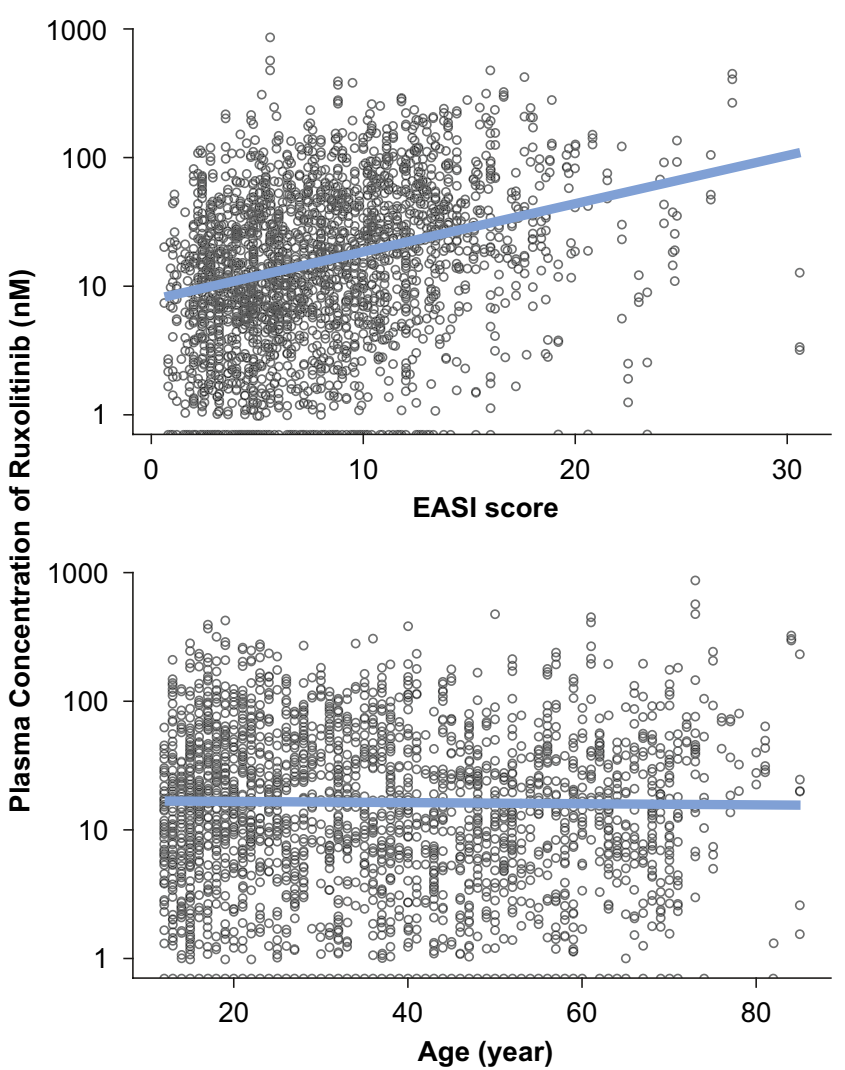

Fig. 3 Steady-state concentration of ruxolitinib, by covariates of potential interest in phase III studies combined. API active pharmaceutical ingredient, $B S A$ body surface area, EASI Eczema Area and Severity Index

Fig. 4 Forest plot of effects of covariates of interest on $C_{\mathrm{ss}}$ of ruxolitinib in phase III studies combined. BSA body surface area, $C_{s s}$ steady-state plasma concentration, IGA Investigator's Global Assessment

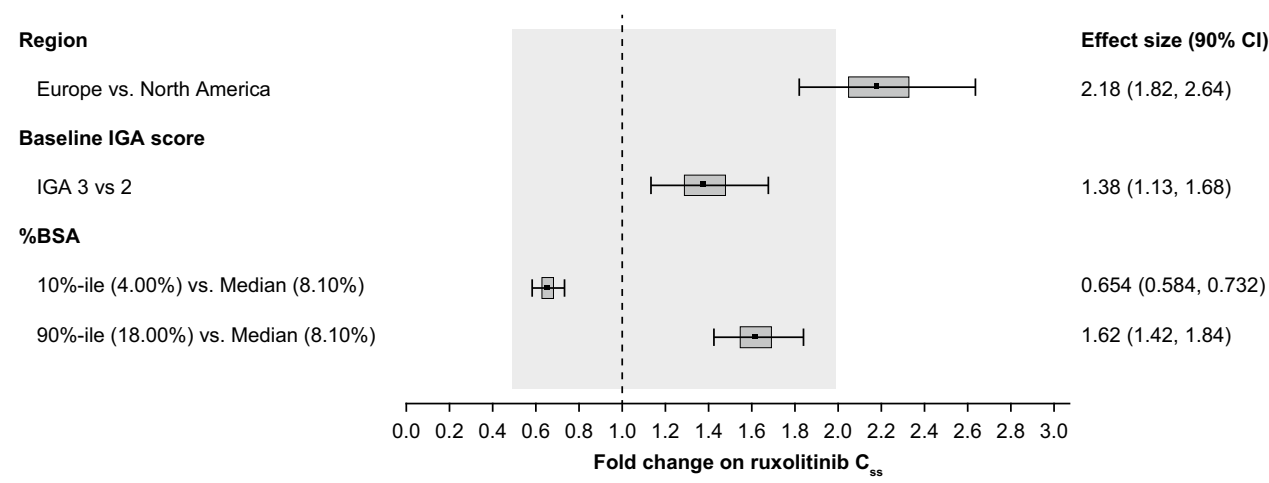


these covariates are 0.782 (90\% CI 0.594-0.970), 0.322 (90\% CI 0.123-0.522), and 0.602 (90\% CI 0.436-0.767), respectively (Table 2 ). The ruxolitinib $C_{\mathrm{ss}}$ showed a lessthan-proportional relationship to amount/dose applied (treatment area) (Table 1, Table 2, and Fig. 4) with the exponent for dose being 0.462 (90\% CI 0.356-0.567). The magnitudes of the covariates' effects are shown in a forest plot in Fig. 4.

There was no apparent difference in the systemic bioavailability between the two different strengths of ruxolitinib formulations used in the phase III studies (7.68\% and 6.22\%, respectively, for $0.75 \% \mathrm{BID}$ and $1.5 \%$ BID) (Table 1). Also, no apparent differences were observed in the bioavailability across the range of \%BSA treated or between the strengths or the two regions (Fig. 5).

\subsection{Pharmacokinetic/Pharmacodynamic Values for Key Hematological Parameters}

An attempt was made to correlate ruxolitinib $C_{\mathrm{ss}}$ with the three key hematological parameters: absolute neutrophil counts, hemoglobin, and platelets. There was no correlation between ruxolitinib $C_{\mathrm{ss}}$ quartiles and hemoglobin or absolute neutrophil count values; patients with ruxolitinib $C_{\mathrm{ss}}>75$ th percentiles did not show any clinically relevant changes in these two hematological parameters (Online Resources 1 and 2, see electronic supplementary material [ESM]). There was a transient increase in platelet count at week 2 with a spontaneous return to baseline by week 4 , which was the next observation period after week 2 . Such increases were minor in scale ( $<30 \%$ change from baseline) and generally within the normal ranges for platelet counts. The change in platelet count at week 2 was dose- and ruxolitinib $C_{\mathrm{ss}^{-}}$ dependent (Fig. 6). The relationships between platelet counts at weeks 4 and 8 and ruxolitinib $C_{\mathrm{ss}}$ were flat. No change was observed in the mean platelet volume (Online Resource 3, see ESM).

\section{Discussion}

Efficacy and safety of treatment with ruxolitinib cream in adolescent and adult patients with atopic dermatitis with cream strengths up to $1.5 \%$ BID were reported in separate publications for the phase II trial $[43,44]$ and the two phase III studies $[46,50]$. The plasma PK profiles after topical ruxolitinib treatment from the phase II and 3 studies are reported here.

The phase II study indicated dose-dependent efficacy and no significant treatment-emergent AEs suggestive of a systemic JAK1/JAK2 inhibition. The PK data from the phase II study were instrumental in understanding the effect of dose and schedule dependency on pharmacokinetics as well as providing guidance on expected plasma concentrations
Fig. 5 Bioavailability versus $\%$ BSA treated by treatment arm and geographical region in phase III studies combined. BID twice daily, $B S A$ body surface area

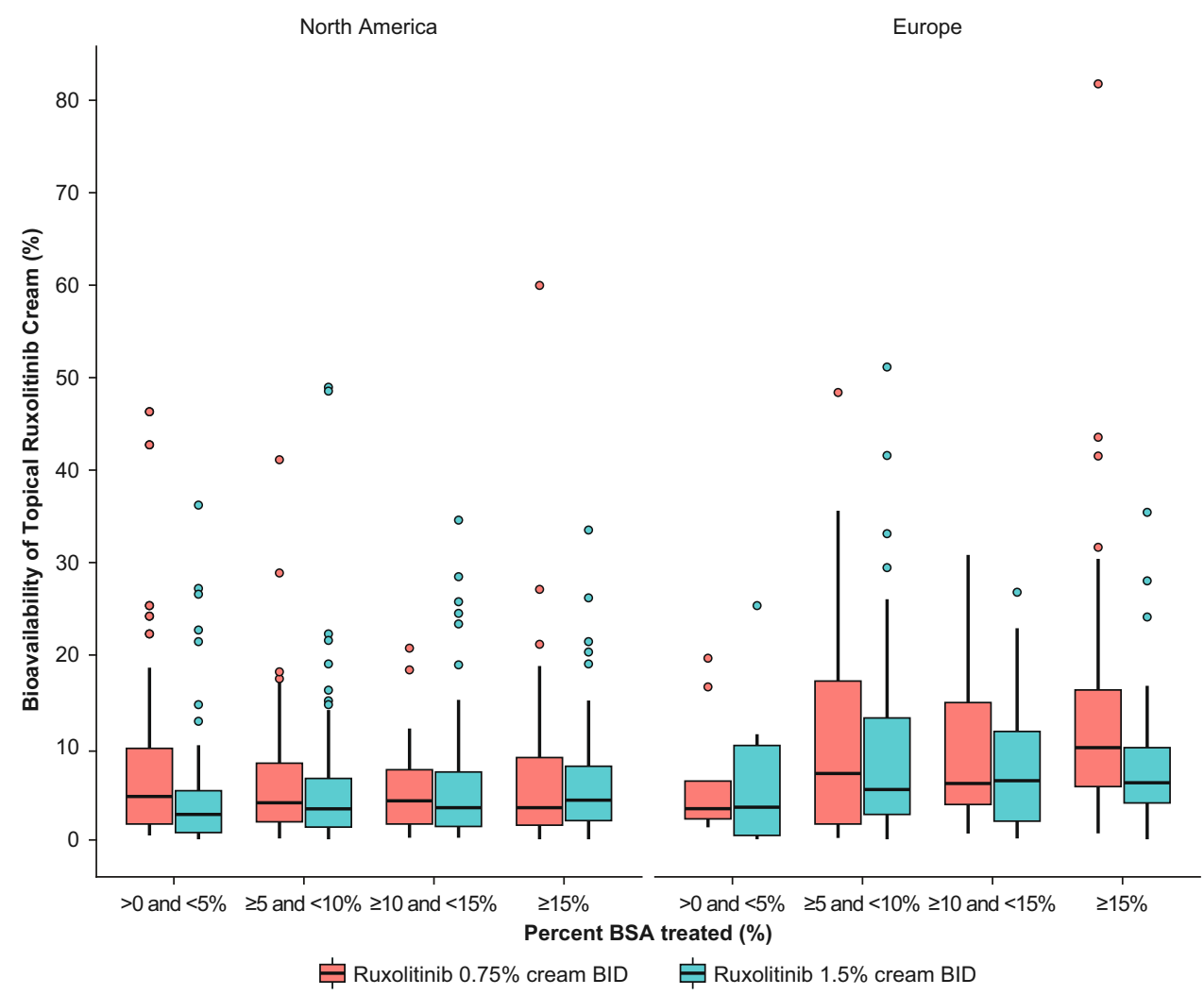


Fig. 6 Mean (95\% CI) platelet count over time after application of ruxolitinib cream in the phase III studies combined. $B Q L$ ruxolitinib cream treatment but PK samples below the quantification limit, $C_{s s}$ steady-state concentration, $Q 1$ to $Q 41$ st to 4th quartiles of ruxolitinib $C_{s s}$ during VC period, $P K$ pharmacokinetic, $V C$ vehicle-controlled

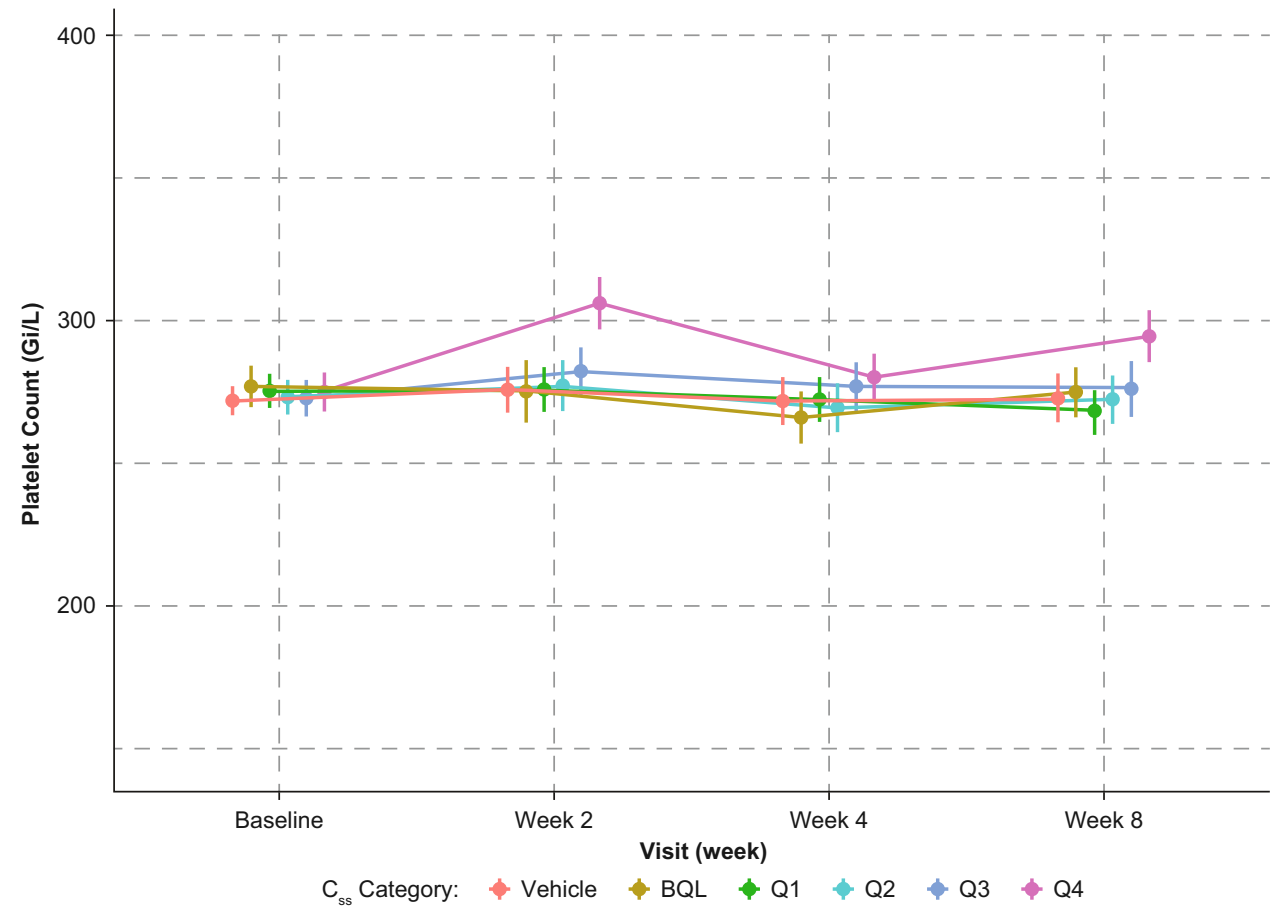

in the phase III studies. Mean plasma concentrations were $4.11 \mathrm{nM}$ at the lowest dose of $0.15 \% \mathrm{QD}$ and $47.7 \mathrm{nM}$ at the highest dose of $1.5 \% \mathrm{BID}$, that is, an approximate 10-fold increase with a 20-fold increase in dose applied, suggesting a markedly sublinear dose versus plasma concentration relationship. The reason for such a sublinear relationship can be related to the release profile of ruxolitinib from the cream formulation and can indicate a transition from a first-order release of ruxolitinib from the stratum corneum to a zeroorder release in an asymptotic manner, from the lowest to highest dose applied, that is, forming a depot at the higher dose. A simple linear regression of data from the three QD treatments provided an estimate of regression slope of 0.805 , indicative of a modestly sublinear increase in plasma concentrations of ruxolitinib with escalating QD doses over the range of $0.15-1.5 \%$. The mean topical bioavailability at the highest topical dose was $5.68 \% \pm 5.58 \%$, which is, expectedly, substantially lower than the near-complete bioavailability estimated after oral dosing [48]. The relatively high interpatient variability around the median plasma concentrations (Fig. 1) and the estimated bioavailability are also to be expected for a product with low bioavailability (e.g., $<10 \%$ ) [51], regardless of route of administration. More importantly, the mean steady-state $C_{\text {trough }}$ at the highest dose of $1.5 \%$ BID is $<20 \%$ of the ruxolitinib half-maximal inhibitory concentration $\left(\mathrm{IC}_{50}\right)$ for JAK2 inhibition in human whole blood assays [52], and this indicates a low probability of any systemic adverse effects associated with JAK1/JAK2 inhibition, such as bone marrow suppression. Overall, the plasma PK profile of ruxolitinib cream in the atopic dermatitis phase II study is similar to that observed in psoriasis patients [53].

The two phase III studies were identical in terms of inclusion and exclusion criteria and the demographics and baseline characteristics were similar as well. Therefore, all analyses reported here are from pooled data from both studies. The mean $C_{\text {trough }}$ through the VC period for the $0.75 \%$ BID and $1.5 \%$ BID doses were 23.8 and $35.7 \mathrm{nM}$, respectively, that is, a less-than-proportional increase in plasma concentrations with a 2-fold increase in the formulation strength, which was similar to the dose versus plasma concentration relationship observed in the phase II study. A simple linear regression provided an estimate for the exponent to be 0.801 , which was similar to that obtained in the phase II study (0.805), in which the regression included three different QD doses versus two different BID doses in the phase III study. The mean bioavailability of ruxolitinib after topical administration of ruxolitinib cream was similar for the two doses $(7.68 \%$ and $6.22 \%$, respectively, for $0.75 \%$ and $1.5 \%$ BID) in the phase III studies, similar to the range observed in phase II studies for the various doses (Table 1). It is interesting to note that the mean bioavailability for ruxolitinib $1.5 \%$ BID in the phase III studies $(6.22 \%)$ was marginally higher compared with that in the phase II study $(5.68 \%)$ despite a lower mean steady-state trough plasma concentration (35.7 nM vs $47.7 \mathrm{nM})$. This is likely related to differences in the drug product application rate $\left(1.47 \mathrm{mg} / \mathrm{cm}^{2}\right.$ in the phase III studies vs $1.92 \mathrm{mg} / \mathrm{cm}^{2}$ in the phase II study). It is possible that the higher drug product application rate in 
the phase II study is related to dispensing of excess product tubes in that study.

Besides the amount of the API used, the other parameter that was expected to influence plasma concentrations of ruxolitinib was \%BSA treated with study drug product. The effect sizes for 90 th or 10th percentile for $\%$ BSA were $>2$-fold change in $C_{\mathrm{ss}}$ compared with the median \%BSA (Fig. 5), demonstrating the impact of \%BSA on plasma concentrations of ruxolitinib. Analysis of the time course of plasma concentrations of ruxolitinib indicates that in the phase III studies, mean ruxolitinib $\mathrm{C}_{\text {trough }}$ were similar at 2, 4, and 8 weeks for each of the dose groups (Fig. 1). This is to be expected as patients were instructed to treat the entire baseline lesion area for 8 weeks, which represents the VC treatment period. Subsequent to week 8 , the mean $C_{\text {trough }}$ were lower than those observed up to week 8 but steady from weeks 12 to 52 . The mean $C_{\text {trough }}$ during the long-term safety (LTS) period were lower than those of the VC period, likely driven by the decrease in amount of product applied, which in turn is due to decreases in affected BSA beyond the 8 weeks of the VC period. In the LTS phase of the protocol, patients are instructed to only apply the cream to active lesions, and therefore any resolution of lesions over the first 8 weeks would result in correspondingly less cream applied. Furthermore, at the time of blood sampling during the LTS period, some patients may have been in remission (off treatment), while others were on active therapy.

An attempt was made to stratify the plasma concentration data based on the two stratification factors used in the phase III studies: geographical region (US or Europe) and baseline IGA score (2 or 3) (Fig 2). There is a trend toward a higher plasma concentration in patients with baseline IGA of 3 versus IGA of 2 and for patients in Europe versus the US, and this difference was most pronounced for patients in Europe with baseline IGA of 3. This trend was observed for each of the two strengths and in each of the individual phase III studies as well. Patients with an IGA of 3 are likely to have more extensive areas affected by their atopic dermatitis (and \%BSA) compared with patients with an IGA of 2, and therefore the difference in plasma concentrations between the two groups is not surprising. This is also evident from the covariate analysis, where plasma concentration showed a positive correlation to EASI score as well as to \%BSA (Fig. 3). The apparent regional differences, with an effect size of 2.18 (Fig. 4), seem to arise from the imbalanced distributions of baseline IGA score and \%BSA between North America and Europe. There was an approximately 13:1 ratio of patients with a baseline IGA score of 3 versus 2 in Europe and an approximately 2:1 ratio in North America. A higher proportion of patients with $>15 \%$ BSA was enrolled in the stratum of a baseline IGA score of 3 in Europe (131
[46.5\%] of a subtotal of 282 patients) than the stratum of a baseline IGA score of 3 in North America (77 [17.6\%] of a subtotal of 438 patients) in the pooled phase III data, which is 1.6-fold higher; this difference was consistent with that observed in the individual phase III studies. Baseline IGA score and \%BSA were positively correlated, because patients with a baseline IGA score of 3 tended to have higher \%BSA affected. Therefore, it is likely that the higher plasma concentrations of ruxolitinib in patients in Europe are simply a function of the more severe disease status at baseline relative to patients in North America.

A statistical linear model was developed to quantitatively characterize the relationship between amount of API per application and the $C_{\mathrm{ss}}$ of ruxolitinib, with geographical region, baseline IGA score, and baseline \%BSA identified as significant predictors, whereas inclusions of additional covariates such as baseline EASI score, sex, race, or age did not significantly improve the model fitness.

Treatments with oral JAK inhibitors are associated with AEs, such as thrombocytopenia, which are believed to be on-target for certain hematological conditions such as myelofibrosis, polycythemia vera, etc. [54]. Ruxolitinib, as a potent inhibitor of the JAK1/JAK2 and JAK/STAT pathway, can cause changes in hematological parameters including thrombocytopenia and anemia when dosed (up to $25 \mathrm{mg}$ BID) orally in patients with myelofibrosis who have compromised bone marrow function $[55,56]$. The maximum tolerated dose in patients with myelofibrosis, who suffer from compromised bone marrow function, is $25 \mathrm{mg}$ BID (oral) [57], which corresponds to an average $C_{\mathrm{ss}}$ of $\sim 350 \mathrm{nM}$ [58], whereas the same dose and the corresponding plasma ruxolitinib concentrations were well tolerated in a healthy volunteer study [48]; in other words, subjects with normal bone marrow function are less susceptible to bone marrow suppression at a given dose or average plasma concentration of ruxolitinib. As a rule of thumb, the potential for bone marrow suppression increases when average plasma concentrations exceed the whole blood $\mathrm{IC}_{50}$ for JAK2 inhibition for several hours/ days in a repeated fashion over the course of at least 2 weeks. For ruxolitinib, this $\mathrm{IC}_{50}$ value is $281 \mathrm{nM}$ [52]. Any changes in hematological parameters of patients with atopic dermatitis after topical administration of ruxolitinib are therefore not expected, given that the mean $C_{\mathrm{ss}}$ are $<$ $40 \mathrm{nM}$, i.e., $<15 \%$ of the $\mathrm{IC}_{50}$ value.

Consistent with this expectation, no clinically relevant changes in hematological parameters were observed in any of the three studies in atopic dermatitis, and therefore correlation analyses could not be generated. The only hematological change observed in the topical ruxolitinib studies was a transient and minor increase in platelets at week 2 with spontaneous (while on treatment) normalization by the next visit at week 4 (Fig. 6), and this change was more 
perceptible for the fourth quartile of ruxolitinib $\mathrm{C}_{\mathrm{ss}}$. Platelet counts remained within normal limits at all visits, including week 2 , and the mechanism behind this transient and modest increase in platelets at week 2 is not clear. However, given that there was no change in the mean platelet volume (indicating a lack of increase in young platelets; Online Resource 3 , see ESM), the transient increase in platelets at week 2 may relate to an activity that is not associated with an effect on bone marrow.

\section{Conclusion}

In conclusion, the PK results from the studies in patients with atopic dermatitis with BSA $\leq 20 \%$ indicate that the overall bioavailability of ruxolitinib cream is low (a fraction of that of the oral formulation) and that the average steady-state trough plasma concentrations at its highest strength (1.5\% cream BID) are well below that required for clinically relevant systemic pharmacological activity. Consistent with the observed plasma concentrations and the expectations thereof, ruxolitinib cream was well tolerated and systemic safety concerns associated with oral JAK inhibitors were not observed in these three studies. The reported results lend support to the emerging profile of ruxolitinib cream as a promising treatment modality in atopic dermatitis.

Supplementary Information The online version contains supplementary material available at https://doi.org/10.1007/s40257-021-00610-x.

Acknowledgements The authors wish to thank the study participants and their families, the investigators, and the site personnel who conducted this study. The authors thank May Venturanza, Brad Yuska, and Susan Lockhead for their work in support of the ruxolitinib cream clinical development program. Editorial assistance was provided by Envision Pharma Group, Inc. (Philadelphia, PA, USA), and funded by Incyte Corporation.

\section{Declarations}

Funding The study was funded by Incyte Corporation, Wilmington, DE, USA.

Conflicts of interest All authors are employees and shareholders of Incyte Corporation.

Ethics approval The phase II and phase III study protocols were approved by the independent ethics committee or institutional review board at each study site, and the studies were conducted in accordance with the ethical principles of the Declaration of Helsinki, International Council for Harmonization E6 Good Clinical Practice consolidated guidelines, and all applicable local laws/regulations.

Consent to participate All patients provided written informed consent before initiating treatment.
Consent for publication Not applicable.

Availability of data and material Incyte Corporation (Wilmington, DE, USA) is committed to data sharing that advances science and medicine while protecting patient privacy. Qualified external scientific researchers may request anonymized datasets owned by Incyte for the purpose of conducting legitimate scientific research. Researchers may request anonymized datasets from any interventional study (except phase I studies) for which the product and indication have been approved on or after 1 January 2020 in at least one major market (e.g., US, EU, JPN). Data will be available for request after the primary publication or 2 years after the study has ended. Information on Incyte's clinical trial data sharing policy and instructions for submitting clinical trial data requests are available at: https://www.incyte.com/Portals/0/Assets/ Compliance\%20and\%20Transparency/clinical-trial-data-sharing.pdf? ver $=2020-05-21-132838-960$

Code availability Not applicable.

Authors' contributions Xiaohua Gong, Xuejun Chen, Michael E. Kuligowski, and Swamy Yeleswaram were involved in the conception/ design of the work. Michael E. Kuligowski, Evan Cimino, and Ryan McGee acquired the data. Xiaohua Gong, Xing Liu, and Xiang Liu analyzed the data. All authors drafted the manuscript or revised it critically for important intellectual content, and approved the manuscript.

Open Access This article is licensed under a Creative Commons Attribution-NonCommercial 4.0 International License, which permits any non-commercial use, sharing, adaptation, distribution and reproduction in any medium or format, as long as you give appropriate credit to the original author(s) and the source, provide a link to the Creative Commons licence, and indicate if changes were made. The images or other third party material in this article are included in the article's Creative Commons licence, unless indicated otherwise in a credit line to the material. If material is not included in the article's Creative Commons licence and your intended use is not permitted by statutory regulation or exceeds the permitted use, you will need to obtain permission directly from the copyright holder. To view a copy of this licence, visit http://creativecommons.org/licenses/by-nc/4.0/.

\section{References}

1. Eichenfield LF, Tom WL, Chamlin SL, Feldman SR, Hanifin JM, Simpson EL, et al. Guidelines of care for the management of atopic dermatitis: section 1. Diagnosis and assessment of atopic dermatitis. J Am Acad Dermatol. 2014;70:338-51. https://doi.org/ 10.1016/j.jaad.2013.10.010.

2. Vinding GR, Zarchi K, Ibler KS, Miller IM, Ellervik C, Jemec GB. Is adult atopic eczema more common than we think?-a population-based study in Danish adults. Acta Derm Venereol. 2014;94:480-2. https://doi.org/10.2340/00015555-1761.

3. Rönmark EP, Ekerljung L, Lötvall J, Wennergren G, Rönmark E, Torén K, et al. Eczema among adults: prevalence, risk factors and relation to airway diseases Results from a large-scale population survey in Sweden. Br J Dermatol. 2012;166:1301-8. https://doi. org/10.1111/j.1365-2133.2012.10904.x.

4. Harrop J, Chinn S, Verlato G, Olivieri M, Norbäck D, Wjst M, et al. Eczema, atopy and allergen exposure in adults: a populationbased study. Clin Exp Allergy. 2007;37:526-35. https://doi.org/ 10.1111/j.1365-2222.2007.02679.x. 
5. Hanifin JM, Reed ML. A population-based survey of eczema prevalence in the United States. Dermatitis. 2007;18:82-91. https:// doi.org/10.2310/6620.2007.06034.

6. Hay RJ, Johns NE, Williams HC, Bolliger IW, Dellavalle RP, Margolis DJ, et al. The global burden of skin disease in 2010: an analysis of the prevalence and impact of skin conditions. J Invest Dermatol. 2014;134:1527-34. https://doi.org/10.1038/jid.2013. 446.

7. Chiesa Fuxench ZC, Block JK, Boguniewicz M, Boyle J, Fonacier L, Gelfand JM, et al. Atopic dermatitis in America study: a crosssectional study examining the prevalence and disease burden of atopic dermatitis in the US adult population. J Invest Dermatol. 2019;139:583-90. https://doi.org/10.1016/j.jid.2018.08.028.

8. Odhiambo JA, Williams HC, Clayton TO, Robertson CF, Asher MI, Group IPTS. Global variations in prevalence of eczema symptoms in children from ISAAC Phase Three. J Allergy Clin Immunol. 2009;124:1251-1258.e23. https://doi.org/10.1016/j.jaci.2009. 10.009 .

9. Silverberg JI. Public health burden and epidemiology of atopic dermatitis. Dermatol Clin. 2017;35:283-9. https://doi.org/10. 1016/j.det.2017.02.002.

10. Silverberg JI, Garg NK, Paller AS, Fishbein AB, Zee PC. Sleep disturbances in adults with eczema are associated with impaired overall health: a US population-based study. J Invest Dermatol. 2015;135:56-66. https://doi.org/10.1038/jid.2014.325.

11. Silverberg JI, Simpson EL. Associations of childhood eczema severity: a US population-based study. Dermatitis. 2014;25:10714. https://doi.org/10.1097/DER.0000000000000034.

12. Spergel JM. Epidemiology of atopic dermatitis and atopic march in children. Immunol Allergy Clin N Am. 2010;30:269-80. https://doi.org/10.1016/j.iac.2010.06.003.

13. Spergel JM. From atopic dermatitis to asthma: the atopic march. Ann Allergy Asthma Immunol. 2010;105:99-106. https://doi.org/ 10.1016/j.anai.2009.10.002 (quiz 7-9).

14. Ellis CN, Drake LA, Prendergast MM, Abramovits W, Boguniewicz M, Daniel CR, et al. Cost of atopic dermatitis and eczema in the United States. J Am Acad Dermatol. 2002;46:361-70. https:// doi.org/10.1067/mjd.2002.120528.

15. Capucci S, Hahn-Pedersen J, Vilsbøll A, Kragh N. Impact of atopic dermatitis and chronic hand eczema on quality of life compared with other chronic diseases. Dermatitis. 2020;31:178-84. https://doi.org/10.1097/der.0000000000000598.

16. Silverberg JI, Gelfand JM, Margolis DJ, Boguniewicz M, Fonacier $\mathrm{L}$, Grayson $\mathrm{MH}$, et al. Patient burden and quality of life in atopic dermatitis in US adults: a population-based cross-sectional study. Ann Allergy Asthma Immunol. 2018;121:340-7. https://doi.org/ 10.1016/j.anai.2018.07.006.

17. Feldman SR, Cox LS, Strowd LC, Gerber RA, Faulkner S, Sierka $\mathrm{D}$, et al. The challenge of managing atopic dermatitis in the United States. Am Health Drug Benefits. 2019;12:83-93.

18. Koszorú K, Borza J, Gulacsi L, Sárdy M. Quality of life in patients with atopic dermatitis. Cutis. 2019;104:174-7.

19. Hebert AA, Stingl G, Ho LK, Lynde C, Cappelleri JC, Tallman AM, et al. Patient impact and economic burden of mild-to-moderate atopic dermatitis. Curr Med Res Opin. 2018;34:2177-85. https://doi.org/10.1080/03007995.2018.1498329.

20. Eichenfield LF, Tom WL, Berger TG, Krol A, Paller AS, Schwarzenberger K, et al. Guidelines of care for the management of atopic dermatitis: section 2. Management and treatment of atopic dermatitis with topical therapies. J Am Acad Dermatol. 2014;71:116-32. https://doi.org/10.1016/j.jaad.2014.03.023.

21. National Eczema Association. Prescription topical treatment. National Eczema Association, Novato, CA, USA. 2020. https:// nationaleczema.org/eczema/treatment/topicals/. Accessed 12 May 2020.
22. Reda AM, Elgendi A, Ebraheem AI, Aldraibi MS, Qari MS, Abdulghani MMR, et al. A practical algorithm for topical treatment of atopic dermatitis in the Middle East emphasizing the importance of sensitive skin areas. J Dermatolog Treat. 2019;30:366-73. https://doi.org/10.1080/09546634.2018.15248 23.

23. Wollenberg A, Barbarot S, Bieber T, Christen-Zaech S, Deleuran M, Fink-Wagner A, et al. Consensus-based European guidelines for treatment of atopic eczema (atopic dermatitis) in adults and children: part I. J Eur Acad Dermatol Venereol. 2018;32:657-82. https://doi.org/10.1111/jdv.14891.

24. Chow S, Seow CS, Dizon MV, Godse K, Foong H, Chan V, et al. A clinician's reference guide for the management of atopic dermatitis in Asians. Asia Pac Allergy. 2018;8:e41. https://doi.org/ 10.5415/apallergy.2018.8.e41.

25. Saeki H, Nakahara T, Tanaka A, Kabashima K, Sugaya M, Murota $\mathrm{H}$, et al. Clinical practice guidelines for the management of atopic dermatitis 2016. J Dermatol. 2016;43:1117-45. https://doi.org/10. 1111/1346-8138.13392.

26. Ring J, Alomar A, Bieber T, Deleuran M, Fink-Wagner A, Gelmetti $\mathrm{C}$, et al. Guidelines for treatment of atopic eczema (atopic dermatitis) part I. J Eur Acad Dermatol Venereol. 2012;26:104560. https://doi.org/10.1111/j.1468-3083.2012.04635.x.

27. Ring J, Alomar A, Bieber T, Deleuran M, Fink-Wagner A, Gelmetti C, et al. Guidelines for treatment of atopic eczema (atopic dermatitis) Part II. J Eur Acad Dermatol Venereol. 2012;26:117693. https://doi.org/10.1111/j.1468-3083.2012.04636.x.

28. Saeki H, Furue M, Furukawa F, Hide M, Ohtsuki M, Katayama I, et al. Guidelines for management of atopic dermatitis. J Dermatol. 2009;36:563-77. https://doi.org/10.1111/j.1346-8138.2009. 00706.x.

29. Pfizer Labs. EUCRISA- crisaborole ointment-Pfizer Laboratories Div Pfizer Inc. Pfizer Inc., New York, NY, USA. 2020. http:// labeling.pfizer.com/ShowLabeling.aspx id=5331. Accessed 12 May 2020.

30. Rodrigues MA, Torres T. JAK/STAT inhibitors for the treatment of atopic dermatitis. J Dermatolog Treat. 2020;31:33-40. https:// doi.org/10.1080/09546634.2019.1577549.

31. Solimani F, Meier K, Ghoreschi K. Emerging topical and systemic JAK inhibitors in dermatology. Front Immunol. 2019;10:2847. https://doi.org/10.3389/fimmu.2019.02847.

32. Howell MD, Kuo FI, Smith PA. Targeting the Janus kinase family in autoimmune skin diseases. Front Immunol. 2019;10:2342. https://doi.org/10.3389/fimmu.2019.02342.

33. Welsch K, Holstein J, Laurence A, Ghoreschi K. Targeting JAK/ STAT signalling in inflammatory skin diseases with small molecule inhibitors. Eur J Immunol. 2017;47:1096-107. https://doi. org/10.1002/eji.201646680.

34. Rerknimitr P, Otsuka A, Nakashima C, Kabashima K. The etiopathogenesis of atopic dermatitis: barrier disruption, immunological derangement, and pruritus. Inflamm Regen. 2017;37:14. https://doi.org/10.1186/s41232-017-0044-7.

35. Brunner PM, Guttman-Yassky E, Leung DY. The immunology of atopic dermatitis and its reversibility with broad-spectrum and targeted therapies. J Allergy Clin Immunol. 2017;139:S65-s76. https://doi.org/10.1016/j.jaci.2017.01.011.

36. Gandhi NA, Bennett BL, Graham NM, Pirozzi G, Stahl N, Yancopoulos GD. Targeting key proximal drivers of type 2 inflammation in disease. Nat Rev Drug Discov. 2016;15:35-50. https://doi.org/ $10.1038 / \mathrm{nrd} 4624$.

37. Bao L, Zhang H, Chan LS. The involvement of the JAK-STAT signaling pathway in chronic inflammatory skin disease atopic dermatitis. Jakstat. 2013;2:e24137. https://doi.org/10.4161/jkst. 24137. 
38. Hashimoto T, Sakai K, Sanders KM, Yosipovitch G, Akiyama T. Antipruritic effects of Janus kinase inhibitor tofacitinib in a mouse model of psoriasis. Acta Derm Venereol. 2019;99:298303. https://doi.org/10.2340/00015555-3086.

39. Levy LL, Urban J, King BA. Treatment of recalcitrant atopic dermatitis with the oral Janus kinase inhibitor tofacitinib citrate. J Am Acad Dermatol. 2015;73:395-9. https://doi.org/10.1016/j. jaad.2015.06.045.

40. Nakagawa $\mathrm{H}$, Nemoto O, Yamada H, Nagata T, Ninomiya N. Phase 1 studies to assess the safety, tolerability and pharmacokinetics of JTE-052 (a novel Janus kinase inhibitor) ointment in Japanese healthy volunteers and patients with atopic dermatitis. J Dermatol. 2018;45:701-9. https://doi.org/10.1111/1346-8138. 14322.

41. Nakagawa H, Nemoto O, Igarashi A, Nagata T. Efficacy and safety of topical JTE-052, a Janus kinase inhibitor, in Japanese adult patients with moderate-to-severe atopic dermatitis: a phase II, multicentre, randomized, vehicle-controlled clinical study. $\mathrm{Br} \mathrm{J}$ Dermatol. 2018;178:424-32. https://doi.org/10.1111/bjd.16014.

42. Bissonnette R, Papp KA, Poulin Y, Gooderham M, Raman M, Mallbris L, et al. Topical tofacitinib for atopic dermatitis: a phase IIa randomized trial. Br J Dermatol. 2016;175:902-11. https://doi. org/10.1111/bjd.14871.

43. Kim BS, Howell MD, Sun K, Papp K, Nasir A, Kuligowski ME. Treatment of atopic dermatitis with ruxolitinib cream (JAK1/ JAK2 inhibitor) or triamcinolone cream. J Allergy Clin Immunol. 2020;145:572-82. https://doi.org/10.1016/j.jaci.2019.08.042.

44. Kim BS, Sun K, Papp K, Venturanza M, Nasir A, Kuligowski ME. Effects of ruxolitinib cream on pruritus and quality of life in atopic dermatitis: results from a phase 2 , randomized, dose-ranging, vehicle- and active-controlled study. J Am Acad Dermatol. 2020;82:1305-13. https://doi.org/10.1016/j.jaad.2020.02.009.

45. Incyte Corporation. JAKAFI ruxolitinib tablets-highlights of prescribing information Incyte Corporation, Wilmington, DE, USA. 2020. https://www.jakafi.com/pdf/prescribing-information. pdf. Accessed 19 May 2020.

46. Papp K, Szepietowski JC, Kircik L, Toth D, Eichenfield LF, Leung DYM, et al. Efficacy and safety of ruxolitinib cream for the treatment of atopic dermatitis: results from two phase 3, randomized, double-blind studies. J Am Acad Dermatol. 2021. https://doi.org/ 10.1016/j.jaad.2021.04.085.

47. Purohit VS, Ports WC, Wang C, Riley S. Systemic tofacitinib concentrations in adult patients with atopic dermatitis treated with $2 \%$ tofacitinib ointment and application to pediatric study planning. J Clin Pharmacol. 2019;59:811-20. https://doi.org/10.1002/jcph. 1360.

48. Shi JG, Chen X, McGee RF, Landman RR, Emm T, Lo Y, et al. The pharmacokinetics, pharmacodynamics, and safety of orally dosed INCB018424 phosphate in healthy volunteers. J Clin Pharmacol. 2011;51:1644-54. https://doi.org/10.1177/0091270010 389469.

49. R Core Team. R: a language and environment for statistical computing. R Foundation for Statistical Computing, Vienna, Austria. 2019. https://www.R-project.org/. Accessed 19 Apr 2021.

50. Revolutionizing Atopic Dermatitis, 5 April 2020. Br J Dermatol. 2020;183:p42. https://doi.org/https://doi.org/10.1111/bjd.19043

51. Daublain P, Feng KI, Altman MD, Martin I, Mukherjee S, Nofsinger R, et al. Analyzing the potential root causes of variability of pharmacokinetics in preclinical species. Mol Pharm. 2017;14:1634-45. https://doi.org/10.1021/acs.molpharmaceut. $6 \mathrm{~b} 01118$.

52. Quintás-Cardama A, Vaddi K, Liu P, Manshouri T, Li J, Scherle $\mathrm{PA}$, et al. Preclinical characterization of the selective JAK1/2 inhibitor INCB018424: therapeutic implications for the treatment of myeloproliferative neoplasms. Blood. 2010;115:3109-17. https://doi.org/10.1182/blood-2009-04-214957.

53. Punwani N, Scherle P, Flores R, Shi J, Liang J, Yeleswaram S, et al. Preliminary clinical activity of a topical JAK1/2 inhibitor in the treatment of psoriasis. J Am Acad Dermatol. 2012;67:658-64. https://doi.org/10.1016/j.jaad.2011.12.018.

54. Newsom M, Bashyam AM, Balogh EA, Feldman SR, Strowd LC. New and emerging systemic treatments for atopic dermatitis. Drugs. 2020;80:1041-52. https://doi.org/10.1007/ s40265-020-01335-7.

55. Verstovsek S, Mesa RA, Gotlib J, Levy RS, Gupta V, DiPersio JF, et al. A double-blind, placebo-controlled trial of ruxolitinib for myelofibrosis. N Engl J Med. 2012;366:799-807. https://doi. org/10.1056/NEJMoa1110557.

56. Cervantes F, Vannucchi AM, Kiladjian JJ, Al-Ali HK, Sirulnik A, Stalbovskaya V, et al. Three-year efficacy, safety, and survival findings from COMFORT-II, a phase 3 study comparing ruxolitinib with best available therapy for myelofibrosis. Blood. 2013;122:4047-53. https://doi.org/10.1182/ blood-2013-02-485888.

57. Verstovsek S, Kantarjian H, Mesa RA, Pardanani AD, CortesFranco J, Thomas DA, et al. Safety and efficacy of INCB018424, a JAK1 and JAK2 inhibitor, in myelofibrosis. N Engl J Med. 2010;363:1117-27. https://doi.org/10.1056/NEJMoa1002028.

58. Chen X, Williams WV, Sandor V, Yeleswaram S. Population pharmacokinetic analysis of orally-administered ruxolitinib (INCB018424 Phosphate) in patients with primary myelofibrosis (PMF), post-polycythemia vera myelofibrosis (PPV-MF) or post-essential thrombocythemia myelofibrosis (PET MF). J Clin Pharmacol. 2013;53:721-30. https://doi.org/10.1002/jcph.102. 